Received: 2021/01/29, Revised: 2021/03/05, Accepted: 2021/03/11, Published: 2021/03/31 @2021 Mio Shimomura et al.; Licence Physical Activity and Nutrition. This is an open access article distributed under the terms of the creative commons attribution license (https:/l creativecommons.org/licenses/by-nc/2.0/), which permits unrestricted use, distribution, and reproduction in any medium, provided the orginal work is properly cited.

${ }^{*}$ Corresponding author : Motoyuki lemitsu, Ph.D.

Faculty of Sport and Health Science, Ritsumeikan University, 1-1-1 Nojihigashi, Kusatsu, Shiga, 525-8577, Japan.

Tel: +81-77-599-4131

Fax: +81-77-561-3761

E-mail: iemitsu@fcritsumei.ac.jp

@2021 The Korean Society for Exercise Nutrition

\section{Relationship between plasma asymmetric dimethylarginine and nitric oxide levels affects aerobic exercise training-induced reduction of arterial stiffness in middle-aged and older adults}

\author{
Mio Shimomura ${ }^{1}$ Shumpei Fujie ${ }^{1}$ / Kiyoshi Sanada ${ }^{1}$ / \\ 1. Faculty of Sport and Health Science, Ritsumeikan University, Shiga, Japan \\ 2. Sports Medicine for Health Promotion, Tokyo Medical University, Tokyo, Japan
} Hiroki Kajimoto ${ }^{1}$ / Takafumi Hamaoka ${ }^{2}$ / Motoyuki Iemitsu ${ }^{1 *}$

\section{INTRODUCTION}

[Purpose] Aerobic exercise training (AT) reverses aging-induced deterioration of arterial stiffness via increased arterial nitric oxide (NO) production. Asymmetric dimethylarginine (ADMA), an endogenous inhibitor of NO synthase, was decreased by AT. However, whether AT-induced changes in ADMA levels are related to changes in nitrite/nitrate (NOx) levels remains unclear. Accordingly, we aimed to clarify whether the relationship between plasma ADMA and NOx levels affected the AT-induced reduction of arterial stiffness in middle-aged and older adults.

[Methods] Thirty-one healthy middle-aged and older male and female subjects ( $66.4 \pm 1.3$ years) were randomly divided into two groups: exercise intervention and sedentary controls. Subjects in the training group completed an 8-week AT (60\%-70\% peak oxygen uptake $\left[\mathrm{VO}_{2 \text { peak }}\right.$ for $45 \mathrm{~min}, 3$ days/week).

[Results] AT significantly increased $\dot{\mathrm{V}}_{\text {2peak }}(P<0.05)$ and decreased carotid $\beta$-stiffness $(P<0.01)$. Moreover, plasma ADMA levels were significantly decreased while plasma NOx levels and NOx/ADMA ratio were significantly increased by AT $(P<0.01)$. Additionally, no sex differences in AT-induced changes of circulating ADMA and NOx levels, NOx/ADMA ratio, and carotid $\beta$-stiffness were observed. Furthermore, the AT-induced increase in circulating ADMA levels was negatively correlated with an increase in circulating NOx levels $(r=$ $-0.414, P<0.05$ ), and the AT-induced increase in NOX/ ADMA ratio was negatively correlated with a decrease in carotid $\beta$-stiffness $(r=-0.514, P<0.01)$.

[Conclusion] These results suggest that the increase in circulating NOx with reduction of ADMA elicited by AT is associated with a decrease in arterial stiffness regardless of sex in middle-aged and older adults.

[Key words] asymmetric dimethylarginine, nitric oxide, exercise training, arterial stiffness, aging, peak oxygen uptake
Alterations in arterial structure and function occur during aging in healthy individuals ${ }^{1}$. An aging-induced decrease in endothelial function leads to deterioration of arterial stiffness ${ }^{2-5}$. This functional deterioration impairs the conduit and buffering functions of arteries, leading to several pathological conditions including hypertension, atherosclerosis, congestive heart failure, stroke, and aortic root regurgitation ${ }^{2-5}$. Several studies have shown that arterial stiffness is lower in physically active individuals than in sedentary individuals $\mathrm{s}^{1,6,7}$. Furthermore, aerobic exercise training (AT) reduces arterial stiffness occurring with advancing age ${ }^{7,8}$. Thus, AT prevents or improves arterial stiffness.

Nitric oxide (NO) is produced from 1-arginine by endothelial NO synthase (eNOS). NO causes vasodilation and inhibits the development of arteriosclerosis and atherosclerosis ${ }^{9}$. Asymmetric dimethylarginine (ADMA), an endogenous inhibitor of NOS, is associated with impaired endothelial function in humans ${ }^{10,11}$. Circulating ADMA levels are negatively correlated with flow-mediated vasodilation (FMD) of the brachial artery in healthy adults and patients ${ }^{12}$. Moreover, clinical evidence suggests that elevated plasma ADMA levels are predictive of cardiovascular events such as coronary artery disease ${ }^{13,14}$, as well as death in patients, and that plasma ADMA levels increase with advancing age ${ }^{15,16}$. Thus, ADMA impairs NO bioavailability in older adults, which may result in enhanced arterial stiffness.

AT ameliorates endothelial dysfunction in older adults, and our previous studies reported that endurance exercise training increased arterial eNOS and phosphorylation, eNOS mRNA, and nitrite/nitrate (NOx) levels with a concomitant improvement of endothelial function in aged rodent models ${ }^{17,18}$. Moreover, in middle-aged and older male and female adults, moderate AT elevated plasma NOx levels and reduced arterial stiffness ${ }^{19}$. Similarly, AT led to decreases in circulating ADMA levels in patients with metabolic syndrome, type 1 diabetes mellitus, coronary and peripheral arterial diseases, obesity, and postmenopausal women ${ }^{20-25}$. Additionally, the AT-induced decrease in circulating ADMA levels is 
correlated with an increase in carotid arterial compliance in postmenopausal women ${ }^{25}$. Therefore, the decrease in circulating ADMA levels by AT might contribute to the improvement of arterial stiffness in postmenopausal women. However, whether the AT-induced decrease in circulating ADMA levels is related to changes in circulating NOx levels remains unclear. The ratio of plasma NOx levels to ADMA levels decreases with advancing age, and the NOx/ ADMA ratio is positively correlated with popliteal FMD in healthy men ${ }^{26}$. Therefore, changes in the NOx/ADMA ratio by AT may be associated with a decrease in arterial stiffness in healthy middle-aged and older male and female adults.

Herein, we aimed to identify whether the relationship between plasma ADMA and NOx levels affected the AT-induced reduction in arterial stiffness. For this, we measured plasma ADMA levels, NOx levels, and arterial stiffness in middle-aged and older male and female adults. Additionally, we examined whether the effect of AT on circulating ADMA levels differed according to sex.

\section{METHODS}

\section{Subjects}

Thirty-one healthy middle-aged and older subjects (total, $\mathrm{n}=31,66.4 \pm 1.3$ years [range, 53-79 years]; male, $\mathrm{n}=13$, $69.8 \pm 1.6$ years [range, 61-79 years]; female, $\mathrm{n}=18,63.9 \pm$ 1.7 years [range, 53-79 years]) volunteered to participate in this study. All volunteers provided written informed consent before participating in the study, which was approved by the Ethics Committee of Ritsumeikan University and was conducted in accordance with the Declaration of Helsinki. This study was registered in the University Hospital Medical Information Network Clinical Trials Registry (UMINCTR, UMIN000035520). All subjects were free from overt signs and symptoms of chronic disease and did not smoke. Subjects taking medications such as anti-hyperlipidemics, anti-hypertensives, or anti-hyperglycemics as well as those with a history of stroke, diabetes, hypertension, hyperlipidemia, cardiac disease, chronic renal failure, and mental disorders were excluded from the study. None of the subjects regularly performed resistance and aerobic exercise. The subjects in this study drank very little alcohol. Since estrogen hormones influence arterial function ${ }^{27}$, we recruited postmenopausal women. Subjects were randomly divided into 2 groups: a training group $(\mathrm{n}=16[$ male $=6 /$ female $=$ $10], 64.8 \pm 2.0$ years $)$ and a control group $(n=15[$ male $=7 /$ female $=8$ ], $68.1 \pm 1.6$ years).

\section{Experimental design}

For all subjects, peak oxygen uptake $\left(\dot{\mathrm{VO}}_{2 \text { peak }}\right)$, body weight, body fat, height, resting systolic blood pressure (SBP), resting diastolic blood pressure (DBP), resting heart rate (HR), resting plasma ADMA concentration, resting plasma NOx concentration, as well as serum concentrations of total cholesterol, high-density lipoprotein (HDL) cholesterol, and triglycerides were measured at the beginning and end of the experiment. Carotid $\beta$-stiffness was examined as an index of arterial stiffness. Before the subjects were tested, they sat quietly for $30 \mathrm{~min}$. Resting brachial SBP, DBP, and HR were measured in duplicate in the supine position using a vascular testing device (OMRON COLIN Co., Tokyo, Japan). At the beginning and end of the study period, fasting blood samples were drawn following at least $48 \mathrm{~h}$ of rest after the last exercise training session. All subjects were instructed not to eat or drink fluids other than water for at least $12 \mathrm{~h}$ prior to blood sampling. In addition, we confirmed that participants did not consume any dietary sources of NOx over the $24 \mathrm{~h}$ prior to testing in either group since NOx can be affected by diet. Thus, both acute effects from the most recent bout of exercise and oral sources of NOx other than NO should be avoided. Serum and plasma samples were immediately centrifuged $\left(1500 \times g, 15 \mathrm{~min}, 4^{\circ} \mathrm{C}\right)$. Blood samples were stored at $-80{ }^{\circ} \mathrm{C}$ until use. Room temperature was maintained at $24^{\circ} \mathrm{C}$ throughout the experiments.

\section{Exercise intervention}

Subjects attended the AT program, which consisted of cycling on a leg ergometer at $60 \%-70 \% \mathrm{VO}_{2 \text { peak }}$ for $45 \mathrm{~min} /$ day, 3 days/week for 8 weeks. For the first two periods of the exercise program, subjects performed at $50 \% \mathrm{VO}_{2 \text { peak }}$ for $40 \mathrm{~min}$. The warm-up and cool-down periods consisted of 5 min of cycling at a $40 \% \mathrm{VO}_{2 \text { peak }}$. Exercise compliance was monitored carefully under direct supervision. Additionally, sedentary control subjects were encouraged to rest during the experimental period.

\section{Measurement of $\mathrm{VO}_{2 \text { peak }}$}

The $\mathrm{VO}_{2 \text { peak }}$ was measured using an incremental cycle exercise test on a cycle ergometer (MINATO, AE-310SRD, Osaka, Japan). The incremental cycle exercise began at a work rate of $60 \mathrm{~W}(30-90 \mathrm{~W})$ for men and $30 \mathrm{~W}(0-60 \mathrm{~W})$ for women. Power output was increased by $15 \mathrm{~W} / \mathrm{min}$ until the subjects could not maintain a fixed pedaling frequency of $60 \mathrm{rpm}$. The subjects were encouraged to exercise at maximum intensity during the ergometer test. HR and rating of perceived exertion (RPE) were monitored minute by minute during the exercise. RPE was obtained using a modified Borg scale. $\mathrm{VO}_{2}$ was monitored during the last $30 \mathrm{~s}$ of each period of the increased work rate. The highest value of $\mathrm{VO}_{2}$ during the exercise test was designated as $\mathrm{VO}_{2 \text { peak }}$ if three out of four of the following criteria were met: (I) a plateau in $\mathrm{VO}_{2}$ with an increase in external work, (II) maximal respiratory exchange ratio $\geq 1.1$, (III) maximal $\mathrm{HR} \geq 90 \%$ of the age-predicted maximum $(208-0.7 \times \text { age })^{28}$, and (IV) $\mathrm{RPE} \geq 18$.

\section{Measurement of the carotid $\beta$-stiffness index}

Carotid $\beta$-stiffness was evaluated as an indicator of arterial stiffness. A combination of ultrasound imaging of the pulsatile common carotid artery and simultaneous applanation of tonometrically obtained arterial pressure from the contralateral carotid artery allowed noninvasive determination of arterial compliance ${ }^{8}$. The carotid artery diameter was measured from images obtained using an ultrasound system equipped with a high-resolution linear array transducer ${ }^{28}$. A 
longitudinal image of the cephalic portion of the common carotid artery was acquired $1-2 \mathrm{~cm}$ proximal to the carotid bulb. All image analyses were performed by the same investigator.

Pressure waveforms and amplitudes were obtained from the common carotid artery using a pencil-shaped probe with a high-fidelity strain gauge transducer (SPT-301; Millar Instruments, Houston, TX, USA $)^{8}$. Because baseline blood pressure levels are subjected to hold-down forces, the pressure signal obtained via tonometry was calibrated by equating the carotid mean arterial blood pressure and DBP to brachial artery values ${ }^{8,29}$. The carotid $\beta$-stiffness index was calculated using the equation $[\ln (\mathrm{P} 1 / \mathrm{P} 0)] /[(\mathrm{D} 1-\mathrm{D} 0) / \mathrm{D} 0]$, where D1 and D0 are the maximum (systolic) and minimum (diastolic) diameters and $\mathrm{P} 1$ and $\mathrm{P} 0$ are the highest (systolic) and lowest (diastolic) blood pressures, respectively.

\section{Measurement of plasma NOx levels}

NOx levels in the plasma were measured using the Griess assay (R\&D Systems, Minneapolis, MN, USA) according to the manufacturer's protocol. All samples were assayed in duplicate. The optical density at $540 \mathrm{~nm}$ was measured using a microplate reader (xMark microplate spectrophotometer; Bio-Rad Laboratories, Hercules, CA, USA). The day-today coefficient of variation of plasma NOx levels was $1.0 \pm$ $0.3 \%$.

\section{Measurement of plasma ADMA levels}

ADMA levels in the plasma were measured using an enzyme-linked immunosorbent assay (ELISA; Immundiagnostik AG, Bensheim, Germany) according to the manufacturer's protocol. All samples were assayed in duplicate. The optical density at $450 \mathrm{~nm}$ was quantified using a microplate reader (xMark microplate spectrophotometer; Bio-Rad Laboratories). The day-to-day coefficient of variation of plasma ADMA levels was $0.6 \pm 0.2 \%$.
Measurements of serum cholesterol and triglyceride levels

Fasting serum levels of total cholesterol, high-density lipoprotein (HDL) cholesterol, and triglycerides were determined using standard enzymatic techniques.

\section{Statistical analysis}

Values are expressed as mean \pm standard errors (SE). The differences between the groups and the two time points were assessed by a two-way repeated-measure analysis of variance followed by Fisher's post hoc test, which was applied when a measurement was significantly different. The relationships between AT-induced changes in plasma ADMA levels and plasma NOx levels or carotid $\beta$-stiffness as well as changes in the NOx/ADMA ratio and carotid $\beta$-stiffness were determined using the Pearson correlation coefficient. Sex differences in the changes in these parameters before and after the AT intervention were compared using a paired Student's t-test. Statistical significance was set at $P<0.05$. All statistical analyses were performed using StatView (5.0, SAS Institute, Tokyo, Japan).

\section{RESULTS}

Comparison of baseline and endpoint characteristics between the training and control groups

Before AT, there were no significant differences in VO2peak between the training and control groups. However, there was a significant interaction between the group with intervention on $\mathrm{VO}_{2 \text { peak }}(P<0.05$, Table 1$)$. Specifically, in the training group, $\mathrm{VO}_{2 \text { peak }}$ significantly increased after AT intervention $(P<0.05$, Table 1$)$. Although there was no significant difference in the carotid $\beta$-stiffness between the training and control groups before the AT, there was a significant interaction of the group with intervention on carotid $\beta$-stiffness $(P<0.01$, Fig 1-A). Carotid $\beta$-stiffness significantly decreased after AT intervention $(P<0.01$, Fig. 1-A). However, no significant differences between the groups or

Table 1. Comparison of characteristics between training and control groups

\begin{tabular}{|c|c|c|c|c|c|c|c|}
\hline & \multicolumn{2}{|c|}{ Control } & \multicolumn{2}{|c|}{ Training } & \multicolumn{3}{|c|}{$p$-value } \\
\hline & Pre & Post & Pre & Post & Group & Time & Interaction \\
\hline Age, years & $68.1 \pm 1.6$ & & $64.8 \pm 2.0$ & & & & \\
\hline Height, cm & $160.6 \pm 2.5$ & $160.3 \pm 2.4$ & $159.0 \pm 2.3$ & $159.1 \pm 2.2$ & 0.548 & 0.978 & 0.940 \\
\hline Body weight, kg & $57.0 \pm 3.2$ & $57.0 \pm 3.3$ & $62.2 \pm 3.0$ & $62.2 \pm 3.0$ & 0.102 & 0.988 & 0.981 \\
\hline $\mathrm{BMI}, \mathrm{kg} / \mathrm{m}^{2}$ & $22.0 \pm 1.0$ & $22.0 \pm 1.0$ & $24.6 \pm 1.1$ & $24.6 \pm 1.1$ & 0.018 & 0.999 & 0.949 \\
\hline $\mathrm{HR}, \mathrm{bpm}$ & $60.0 \pm 2.0$ & $57.2 \pm 2.1$ & $59.0 \pm 1.4$ & $58.3 \pm 2.2$ & 0.977 & 0.375 & 0.590 \\
\hline SBP, $\mathrm{mmHg}$ & $134.1 \pm 3.6$ & $129.3 \pm 4.3$ & $128.6 \pm 4.7$ & $118.8 \pm 3.8$ & 0.047 & 0.092 & 0.493 \\
\hline $\mathrm{DBP}, \mathrm{mmHg}$ & $78.3 \pm 2.0$ & $76.1 \pm 2.3$ & $78.0 \pm 3.0$ & $73.9 \pm 2.9$ & 0.637 & 0.237 & 0.730 \\
\hline Total cholesterol, mg/dl & $230.5 \pm 9.9$ & $219.6 \pm 10.0$ & $213.5 \pm 7.7$ & $219.0 \pm 8.5$ & 0.333 & 0.765 & 0.367 \\
\hline HDL cholesterol, mg/dl & $79.5 \pm 6.5$ & $79.7 \pm 7.0$ & $67.6 \pm 5.1$ & $68.9 \pm 5.5$ & 0.067 & 0.901 & 0.927 \\
\hline Triglycerides, mg/dl & $111.7 \pm 25.3$ & $112.4 \pm 29.5$ & $140.8 \pm 23.6$ & $123.1 \pm 18.0$ & 0.417 & 0.727 & 0.707 \\
\hline $\mathrm{V}_{2 \text { peak }}, \mathrm{ml} / \mathrm{kg} / \mathrm{min}$ & $26.7 \pm 1.7$ & $26.5 \pm 1.5$ & $24.0 \pm 1.3$ & $29.9 \pm 1.5^{*}$ & 0.791 & 0.065 & 0.043 \\
\hline
\end{tabular}

$\mathrm{BMI}$, body mass index; HR, heart rate; SBP, systolic blood pressure; DBP, diastolic blood pressure;

$\mathrm{HDL}$, high-density lipoprotein; $\dot{\mathrm{V}}_{2 \text { peak }}$, peak oxygen uptake

The statistical analysis was used by 2-way ANOVA.

The $P$ values of Group and Time were the main effect while the P values of Interaction were Group $\times$ Time interaction.

Values are mean and SE. ${ }^{*} P<0.05$ vs. Training-Pre. 
times were noted for height, body weight, BMI, HR, SBP, DBP, serum total cholesterol levels, HDL cholesterol levels, or triglyceride levels (Table 1).

Comparison of plasma ADMA and NOx levels and NOx/ADMA ratio between the training and control groups

Before AT, there were no significant differences in plasma ADMA or NOx levels and NOx/ADMA ratio between the training and control groups. There was significant interaction between plasma NOx levels in the intervention group (Fig. 1-B, $P<0.01$ ). After AT, plasma NOx levels were significantly higher in the training group than in the control group (Fig. 1-B, $P<0.01$ ). The interaction between groups and time points was significant for plasma ADMA levels (Fig. 1-C, $P<0.05$ ). After AT, plasma ADMA concentrations were significantly lower in the training group than in the control group (Fig. 1-C, $P<0.01$ ). Notably, there was a significant interaction between the intervention group and the NOx/ADMA ratio (Fig. $1-\mathrm{D}, P<0.01$ ). After AT, the NOx/ADMA ratio was significantly higher in the training group than in the control group (Fig. 1-D, $P<0.01$ ).
A

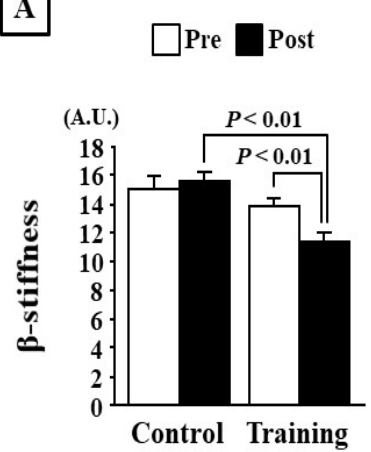

$\mathrm{C}$

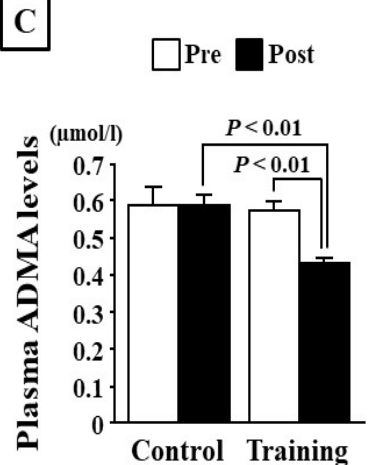

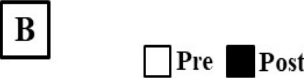

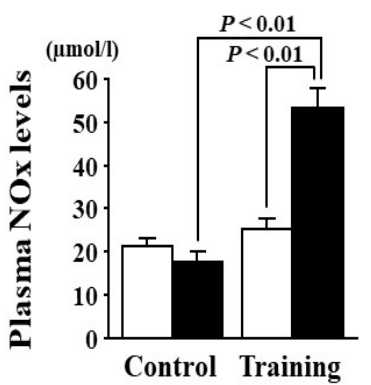

D

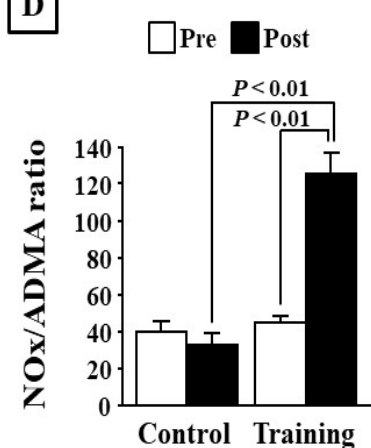

Figure 1. Comparisons of carotid $\beta$-stiffness, plasma nitrite/nitrate (NOx) levels, asymmetric dimethylarginine (ADMA) levels, and NOx ADMA ratio.

Carotid $\beta$-stiffness (A), plasma nitrite/nitrate (NOx: B) levels, asymmetric dimethylarginine (ADMA: C) levels, and NOx/ADMA ratio (D) in middle-aged and older adults before and after 8 weeks of aerobic exercise (training group, $n=16$ ) or sedentary lifestyle (control group, $n$ 15). Open bar: before intervention; solid bar: after intervention. Data are expressed as the mean \pm SE.
Relationships among plasma ADMA and NOx levels, NOx/ADMA ratio, and carotid $\beta$-stiffness

Changes in plasma ADMA levels were negatively correlated with changes in plasma NOx levels (Fig. 2-A, $\mathrm{r}=$ $-0.414, P<0.05)$. Moreover, there was a negative correlation between changes in plasma NOx levels and carotid $\beta$-stiffness (Fig. 2-B, $\mathrm{r}=-0.493, P<0.01$ ). Furthermore, changes in the NOx/ADMA ratio were negatively correlated with changes in carotid $\beta$-stiffness (Fig. 2-C, $\mathrm{r}=-0.514, P<$ $0.01)$.

Sex differences among changes in carotid $\beta$-stiffness, plasma ADMA and NOx levels, and NOx/ADMA ratio

There was no sex difference with regard to changes in carotid $\beta$-stiffness between male and female subjects (Fig. 3-A, male: $-2.400 \pm 0.411$ vs. female: $-2.390 \pm 0.254$ ). In addition, no sex differences were observed in the changes in plasma ADMA and NOx levels or in NOx/ADMA

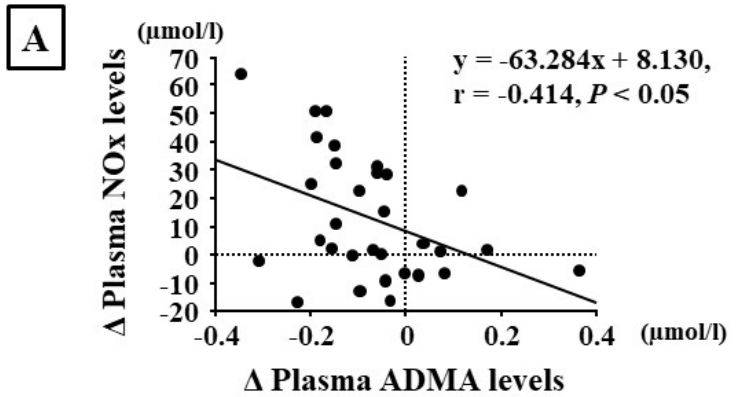

B

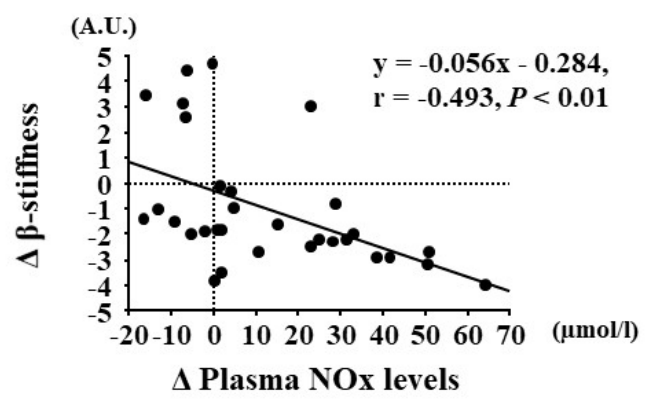

$\mathrm{C}$

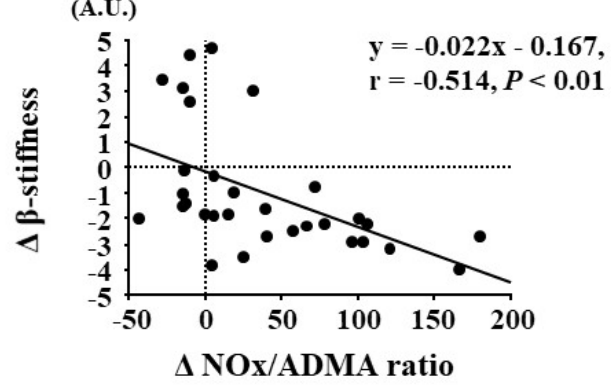

Figure 2. Relationships among changes in plasma asymmetric dimethylarginine (ADMA) and nitrite/nitrate (NOx) levels, NOx/ADMA ratio, and carotid $\beta$-stiffness.

Correlations between the change in plasma nitrite/nitrate (NOx) levels, plasma asymmetric dimethylarginine (ADMA) levels (A), and carotid $\beta$-stiffness (B), as well as the NOx/ADMA ratio and carotid $\beta$-stiffness $(C)$ before and after an intervention in middle-aged and older adults. 
A
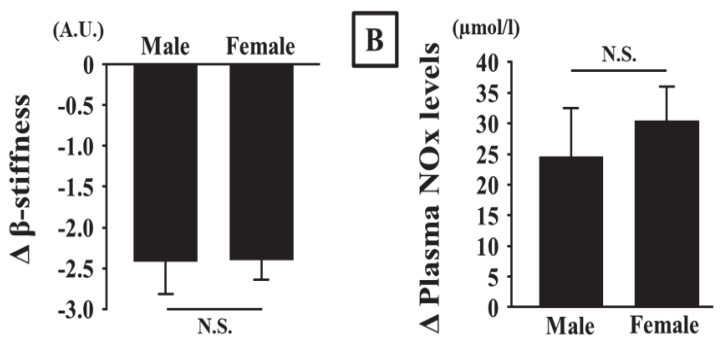

c

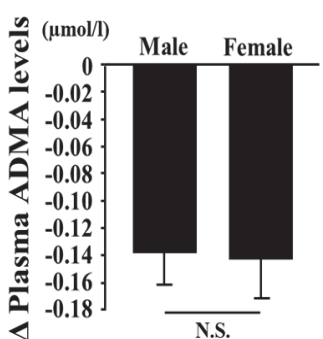

D

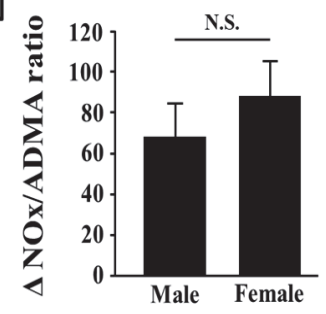

Figure 3. Comparisons of sex differences in changes in carotid $\beta$-stiffness, plasma nitrite/nitrate (NOx) levels, asymmetric dimethylarginine (ADMA) levels, and NOx/ADMA ratio.

Comparison of sex differences in changes in carotid $\beta$-stiffness (A) plasma nitrite/nitrate (NOx: B) levels, asymmetric dimethylarginine (ADMA: C) levels, and NOx/ADMA ratio (D) in middle-aged and older adults before and after 8 weeks of aerobic exercise (training group, $n$ $=16$ ) or sedentary (control group, $n=15$ ). Data are expressed as the mean \pm SE

ratio between male and female subjects (Fig. 3-B, NOx; male: $24.412 \pm 7.997$ vs. female: $30.203 \pm 5.810$, Fig. $3-\mathrm{C}$, ADMA; male: $-0.138 \pm 0.024$ vs. female: $-0.142 \pm 0.029$, Fig. 3-D, NOx/ADMA ratio; male: $67.831 \pm 16.707$ vs. female: $87.964 \pm 17.026$ )

\section{DISCUSSION}

The present study investigated the effects of AT on circulating ADMA levels in middle-aged and older male and female adults before and after an 8-week AT. After AT intervention, plasma ADMA levels and arterial stiffness decreased, and concomitantly, plasma NOx levels and NOx/ ADMA ratio increased. Additionally, we did not observe a sex difference in AT-induced changes in circulating ADMA levels and NOx/ADMA ratio between male and female subjects. Furthermore, the training effect of plasma ADMA levels was negatively correlated with that of plasma NOx levels, and the training effect of NOx/ADMA ratio was negatively correlated with that of carotid $\beta$-stiffness. Thus, this study showed, for the first time, that the changes in the NOx/ADMA ratio from AT were associated with an underlying mechanism of AT-induced improvement in arterial stiffness.

In previous studies, there were no reported sex-dependent differences in circulating ADMA levels in kidney transplant patients ${ }^{30}$ and young patients ${ }^{26}$. It has been reported that AT decreases circulating ADMA levels in postmenopausal women $^{25}$. Notably, this study showed that AT decreased circulating ADMA levels in postmenopausal women as well as in middle-aged and older male adults. Additionally, the amount of change in circulating ADMA levels by AT did not differ between the sexes. Thus, the data indicate that there is no sex difference in the effect of AT on circulating ADMA levels. Moreover, low circulating ADMA levels have been independently associated with increased FMD in subjects with low cardiovascular risk ${ }^{31}$ or decreased carotid-femoral pulse wave velocities in prediabetic individuals ${ }^{32}$. Accordingly, the decreased circulating ADMA levels by AT may be a novel biomarker, regardless of sex, for the prevention or treatment of arterial stiffness associated with impairments in NO production.

We demonstrated that an 8-week AT consisting of cycling on a leg ergometer at $60 \%-70 \% \mathrm{VO}_{2 \text { peak }}$ for $45 \mathrm{~min}, 3$ days/week, decreased plasma ADMA levels in middle-aged and older male and female adults. Similarly, 12 weeks of AT consisting of cycling at $65 \%-80 \%$ of maximal HR $40-60$ min/day, 3-6 days/week, decreases circulating ADMA levels in postmenopausal women ${ }^{25}$. In addition, in patients with coronary artery disease, 12 weeks of endurance training consisting of running or walking for 30-60 min, 3-5 days/ week, decreases circulating ADMA levels ${ }^{23}$. Moreover, in patients with metabolic syndrome, circulating ADMA levels were normalized to healthy control levels after 12 weeks of endurance training consisting of cycling on a leg ergometer at anaerobic threshold exercise intensities for $45 \mathrm{~min}, 3$ days $/$ week $^{20}$, and in patients with peripheral artery disease that had performed intermittent walking for $50 \mathrm{~min}, 2$ days/ week for 12 weeks, circulating ADMA levels decreased as compared to the control group ${ }^{24}$. Furthermore, in patients with type 1 diabetes mellitus, circulating ADMA levels were decreased after a 4-month-long aerobic exercise program of cycling on a leg ergometer at $60 \%-70 \%$ maximum HR for $40 \mathrm{~min}, 3$ days $/$ week $^{22}$. Thus, AT may be effective in reducing plasma ADMA levels and arterial stiffness in both healthy and at-risk subjects.

After AT intervention, plasma ADMA levels decreased, and concomitantly, plasma NOx levels and NOx/ADMA ratio were increased in middle-aged and older adults. The mechanism underlying the effects of AT on plasma ADMA levels is unclear. ADMA is selectively degraded by dimethylarginine dimethylaminohydrolase (DDAH) enzymes ${ }^{33}$. DDAH exists in two isoforms. In an in vitro study using siRNA, individual silencing of either DDAH-1 or DDAH2 reduced endothelial NO production by $31 \%$ and $48 \%$, respectively ${ }^{33}$. These results indicate that both DDAH-1 and DDAH-2 play important roles in regulating endothelial NO production. Additionally, intracellular reactive oxygen species (ROS) can stimulate ADMA production or inhibit ADMA degradation, thus resulting in the accumulation of ADMA in endothelial cells ${ }^{34}$. Furthermore, ROS regulate the ADMA/DDAH-2/eNOS/NO pathway in endothelial cells $^{35}$. AT increases eNOS phosphorylation and NOx levels in the aorta of aged rodent models ${ }^{17,18}$. Therefore, we speculate that AT could be associated with upregulated activity and protein expression of DDAH accompanied by a decrease in ADMA concentrations and an increase in 
NO metabolites, thereby reducing arterial stiffness. Further studies are needed to examine the effects of AT on DDAH activity and protein expression in the arterial wall to clarify the mechanistic association between AT-induced changes in ADMA levels and improvement in arterial stiffness.

In conclusion, we investigated the effects of AT on ADMA levels in middle-aged and older male and female adults before and after 8 weeks of AT. The training effect on plasma ADMA levels was related to that of plasma NOx levels, while the training effect on NOx/ADMA ratio was related to that of arterial stiffness. Thus, AT-induced reduction of plasma ADMA levels, regardless of sex, was related to an AT-induced increase in plasma NOx levels. The balance between ADMA and NO production may be associated with an underlying mechanism of AT-induced improvement in arterial stiffness in middle-aged and older men and women.

\section{ACKNOWLEDGEMENTS}

The authors declare no conflicts of interest. This work was supported by Grants-in-Aid for Scientific Research from the Ministry of Education, Culture, Sports, Science, and Technology of Japan (\#19K22828, M. Iemitsu).

\section{REFERENCES}

1. Vaitkevicius PV, Fleg JL, Engel JH, O'Connor FC, Wright JG, Lakatta LE, Yin FC, Lakatta EG. Effects of age and aerobic capacity on arterial stiffness in healthy adults. Circulation. 1993;88:145662.

2. Arnett DK, Evans GW, Riley WA. Arterial stiffness: a new cardiovascular risk factor? Am J Epidemiol. 1994;140:669-82.

3. Blacher J, Asmar R, Djane S, London GM, Safar ME. Aortic pulse wave velocity as a marker of cardiovascular risk in hypertensive patients. Hypertension. 1999;33:1111-7.

4. Laurent S, Boutouyrie P, Asmar R, I Gautier, B Laloux, L Guize, $P$ Ducimetiere, A Benetos. Aortic stiffness is an independent predictor of all-cause and cardiovascular mortality in hypertensive patients. Hypertension. 2001;37:1236-41.

5. Rowe JW. Clinical consequences of age-related impairments in vascular compliance. Am J Cardiol. 1987;60:68G-71G.

6. Mohiaddin RH, Underwood SR, Bogren HG, Firmin DN, Klipstein $\mathrm{RH}$, Rees RS, Longmore DB. Regional aortic compliance studied by magnetic resonance imaging: the effects of age, training, and coronary artery disease. Br Heart J. 1989;62:90-6.

7. Tanaka H, DeSouza CA, Seals DR. Absence of age-related increase in central arterial stiffness in physically active women. Arterioscler Thromb Vasc Biol. 1998;18:127-32.

8. Tanaka H, Dinenno FA, Monahan KD, Clevenger CM, DeSouza CA, Seals DR. Aging, habitual exercise, and dynamic arterial compliance. Circulation. 2000;102:1270-5.

9. Napoli C, de Nigris F, Williams-Ignarro S, Pignalosa O, Sica V, lgnarro LJ. Nitric oxide and atherosclerosis: an update. Nitric Oxide. 2006;15:265-79.

10. Antoniades $\mathrm{C}$, Shirodaria $\mathrm{C}$, Leeson $\mathrm{P}$, Antonopoulos A, Warrick N,
Van-Assche T, Cunnington C, Tousoulis D, Pillai R, Ratnatunga C, Stefanadis $\mathrm{C}$, Channon KM. Association of plasma asymmetrical dimethylarginine (ADMA) with elevated vascular superoxide production and endothelial nitric oxide synthase uncoupling: implications for endothelial function in human atherosclerosis. Eur Heart $\mathrm{J}$. 2009;30:1142-50.

11. Böger RH. Asymmetric dimethylarginine (ADMA): a novel risk marker in cardiovascular medicine and beyond. Ann Med. 2006;38:126-36.

12. Antoniades $C$, Demosthenous $M$, Tousoulis $D$, Antonopoulos AS, Vlachopoulos C, Toutouza M, Marinou K, Bakogiannis C, Mavragani K, Lazaros G, Koumallos N, Triantafyllou C, Lymperiadis D, Koutsilieris M, Stefanadis C. Role of asymmetrical dimethylarginine in inflammation-induced endothelial dysfunction in human atherosclerosis. Hypertension. 2011;58:93-8.

13. Böger RH, Sullivan LM, Schwedhelm E, Wang TJ, Maas R, Benjamin EJ, Schulze F, Xanthakis V, Benndorf RA, Vasan RS. Plasma asymmetric dimethylarginine and incidence of cardiovascular disease and death in the community. Circulation. 2009;119:1592-600.

14. Schnabel R, Blankenberg S, Lubos E, Lackner KJ, Rupprecht HJ, Espinola-Klein C, Jachmann N, Post F, Peetz D, Bickel C, Cambien F, Tiret L, Münzel T. Asymmetric dimethylarginine and the risk of cardiovascular events and death in patients with coronary artery disease: results from the AtheroGene Study. Circ Res. 2005;97:e53-9.

15. Kielstein JT, Bode-Böger SM, Frölich JC, Ritz E, Haller H, Fliser D. Asymmetric dimethylarginine, blood pressure, and renal perfusion in elderly subjects. Circulation. 2003;107:1891-5.

16. Ngo DT, Sverdlov AL, McNeil JJ, Horowitz JD. Correlates of arterial stiffness in an ageing population: role of asymmetric dimethylarginine. Pharmacol Res. 2009;60:503-7.

17. Tanabe T, Maeda S, Miyauchi T, lemitsu M, Takanashi M, Irukayama-Tomobe Y, Yokota T, Ohmori H, Matsuda M. Exercise training improves ageing-induced decrease in eNOS expression of the aorta. Acta Physiol Scand. 2003;178:3-10.

18. Fujie S, Hasegawa N, Horii N, Inoue K, Uchida M, lemitsu M. Effects of combined exercise training and Chlorella intake on vasorelaxation mediated by nitric oxide in aged mice. Appl Physiol Nutr Metab. In press.

19. Fujie S, Hasegawa N, Sato K, Fujita S, Sanada K, Hamaoka T, lemitsu M. Aerobic exercise training-induced changes in serum adropin level are associated with reduced arterial stiffness in middle-aged and older adults. Am J Physiol Heart Circ Physiol. 2015;309:H1642-7.

20. Gomes VA, Casella-Filho A, Chagas AC, Tanus-Santos JE. Enhanced concentrations of relevant markers of nitric oxide formation after exercise training in patients with metabolic syndrome. Nitric Oxide. 2008;19:345-50.

21. Maeda S, Miyaki A, Kumagai H, Eto M, So R, Tanaka K, Ajisaka $R$. Lifestyle modification decreases arterial stiffness and plasma asymmetric dimethylarginine level in overweight and obese men. Coron Artery Dis. 2013;24:583-8.

22. Mittermayer F, Pleiner J, Krzyzanowska K, Wiesinger GF, Francesconi M, Wolzt M. Regular physical exercise normalizes elevated asymmetrical dimethylarginine concentrations in patients with type 1 diabetes mellitus. Wien Klin Wochenschr. 2005;117:816-20.

23. Richter B, Niessner A, Penka M, Grdić M, Steiner S, Strasser B, Ziegler S, Zorn G, Maurer G, Simeon-Rudolf V, Wojta J, Huber K. 
Endurance training reduces circulating asymmetric dimethylarginine and myeloperoxidase levels in persons at risk of coronary events. Thromb Haemost. 2005;94:1306-11.

24. Schlager O, Giurgea A, Schuhfried O, Seidinger D, Hammer A, Gröger M, Fialka-Moser V, Gschwandtner M, Koppensteiner $\mathrm{R}$, Steiner $\mathrm{S}$. Exercise training increases endothelial progenitor cells and decreases asymmetric dimethylarginine in peripheral arterial disease: a randomized controlled trial. Atherosclerosis. 2011;217:240-8.

25. Tanahashi K, Akazawa N, Miyaki A, Choi Y, Ra SG, Matsubara T, Kumagai H, Oikawa S, Maeda S. Aerobic exercise training decreases plasma asymmetric dimethylarginine concentrations with increase in arterial compliance in postmenopausal women. Am J Hypertens. 2014;27:415-21.

26. Nakamura A, Kajitani S, Sato K, Kanazawa M, Kondo M, Endo H, Nozaki E. Decline of popliteal artery flow-mediated dilation with aging and possible involvement of asymmetric dimethylarginine in healthy men. J Med Ultrason. 2019;46:503-11.

27. Weinberger MH, Fineberg NS, Fineberg SE. Effects of age, race, gender, blood pressure, and estrogen on arterial compliance. $A m$ J Hypertens. 2002;15:358-63.

28. Tanaka H, Monahan KD, Seals DR. Age-predicted maximal heart rate revisited. J Am Coll Cardiol. 2001;37:153-6.

29. Kawano $\mathrm{H}$, Tanaka H, Miyachi M. Resistance training and arterial compliance: keeping the benefits while minimizing the stiffening. $J$ Hypertens. 2006;24:1753-9.

30. Cvetkovic TP, Stefanovic NZ, Velickovic-Radovanovic RM, Paunovic GJ, Djordjevic VM, Stojanovic DR, Stojanovic IR, Pavlovic DD. Sex differences in oxidative and nitrosative stress parameters in kidney transplant patients on tacrolimus-based immunosuppression. Int Urol Nephrol. 2014;46:1217-24.

31. Ardigo D, Stüehlinger M, Franzini L, Valtueña S, Piatti PM, Pachinger $\mathrm{O}$, Reaven GM, Zavaroni I. ADMA is independently related to flow-mediated vasodilation in subjects at low cardiovascular risk. Eur J Clin Investig. 2007;37:263-9.

32. Protopsaltis I, Foussas S, Angelidi A, Gritzapis A, Sergentanis TN, Matsagos S, Tzirogiannis K, Panoutsopoulos GI, Dimitriadis G, Raptis S, Melidonis A. Impact of ADMA, endothelial progenitor cells and traditional cardiovascular risk factors on pulse wave velocity among prediabetic individuals. Cardiovasc Diabetol. 2012;11:141.

33. Pope AJ, Karuppiah K, Cardounel AJ. Role of the PRMT-DDAHADMA axis in the regulation of endothelial nitric oxide production. Pharmacol Res. 2009; 60: 461-5.

34. Chen $Y, X u X$, Sheng M, Zhang X, Gu Q, Zheng Z. PRMT-1 and DDAHs-induced ADMA upregulation is involved in ROS- and RAS-mediated diabetic retinopathy. Exp Eye Res. 2009;89:102834.

35. Chen X, Li H, Wang Z, Zhou Q, Chen S, Yang B, Yin D, He H, He $M$. Quercetin protects the vascular endothelium against iron overload damages via ROS/ADMA/DDAH II /eNOS/NO pathway. Eur J Pharmacol. 2020;868:172885. 